\title{
Cross helicity in stellar magnetoconvection
}

\author{
Manfred Küker and Günther Rüdiger \\ Astrophysikalisches Institut Potsdam, \\ An der Sternwarte 16, 14482, Potsdam, Germany
}

\begin{abstract}
Magnetic diffusion is a key ingredient in mean-field dynamo models but neither observations nor theory are able to produce reliable values. Numerical simulations provide an alternative way to determine the turbulent electromotive force. Cross helicity allows us to determine the turbulent magnetic diffusion coefficient in simulations of stellar magnetoconvection.
\end{abstract}

Keywords. convection, Sun: activity, stars: activity

\section{Introduction}

Mean-field MHD has successfully modelled the solar differential rotation and many aspects of its activity cycle. In this formulation of MHD, the mean magnetic field is governed by the induction equation

$$
\frac{\partial\langle\boldsymbol{B}\rangle}{\partial t}=\nabla \times\left(\langle\boldsymbol{u}\rangle \times\langle\boldsymbol{B}\rangle-\eta \nabla \times\langle\boldsymbol{B}\rangle+\left\langle\boldsymbol{u}^{\prime} \times \boldsymbol{B}^{\prime}\right\rangle\right)
$$

where $\eta$ is the magnetic diffusion coefficient, brackets indicate mean (i.e. averaged) quantities and primes the fluctuations. Eq.1.1 is formally identical with the standard induction equation except for the last term on the right hand side, the turbulent electromotive force. The latter can be expressed in terms of the mean field:

$$
\left\langle\boldsymbol{u}^{\prime} \times \boldsymbol{B}^{\prime}\right\rangle \approx \alpha\langle\boldsymbol{B}\rangle-\eta_{T}\langle\nabla \times \boldsymbol{B}\rangle .
$$

The first term on the RHS is the field-generating $\alpha$ effect caused by kinetic helicity and the second term is turbulent diffusion. While the $\alpha$ effect is caused by the Coriolis force, the turbulent magnetic diffusivity exists in non-rotating turbulence, too. Both the $\alpha$ and $\eta_{T}$ coefficients depend on statistical properties of the fluctuations only.

The kinetic helicity on the solar surface has been measured by Komm et al. (2008). The magnetic diffusivity can be derived from the decay of sunspots. However, these are areas with strong magnetic fields and the diffusivity is quenched by the back-reaction of the magnetic field on the fluid. The magnetic diffusivity for the quiet Sun can be determined by measuring the cross helicity, $\left\langle\boldsymbol{u}^{\prime} \cdot \boldsymbol{B}^{\prime}\right\rangle$. The component involving the radial components of the gas velocity and the magnetic field can be expressed in terms of the radial component of the mean magnetic field (Rüdiger et al. 2011):

$$
\frac{\left\langle u_{r}^{\prime} B_{r}^{\prime}\right\rangle}{\left\langle B_{r}\right\rangle} \simeq-\frac{\eta_{T}}{H_{\rho}},
$$

where $H_{\rho}$ is the density scale height. Eq. 1.3 allows the determination of the turbulent magnetic diffusivity coefficient from measurements of the radial velocity and magnetic field components provided the density stratification is known. 


\section{Numerical simulations}

Rüdiger et al. (2011) studied the cross helicity in forced turbulence and found it to be anti-correlated with the radial magnetic field, as required by Eq. 1.3 for a positive magnetic diffusivity. Here we check if this result holds for convection. We therefore run numerical simulations using the Nirvana code (Ziegler 2002), which uses a conservative finite difference scheme in cartesian coordinates. The code solves the equation of motion, the induction equation, and the equations of energy and mass conservation. We assume an ideal, fully ionized gas that is heated from below and kept at a fixed temperature at the top of the simulation box. Periodic boundary conditions apply at the horizontal boundaries. A homogeneous vertical magnetic field is applied. The upper and lower boundaries are impenetrable and stress-free.

In the dimensionless units described there the size of our simulation box is $6 \times 6 \times 2$ in the $x, y$, and $z$ directions, respectively. As we treat the non-rotating case, the $\mathrm{z}$ axis is aligned with the stratification vector, i.e. represents the radial direction in spherical geometry. The $\mathrm{x}$ and $\mathrm{y}$ coordinates denote the horizontal directions. The stratification of density, pressure, and temperature is piecewise polytropic as described in Ziegler (2002). Similar setups have been used by Cattaneo et al. (1991), Brandenburg et al. (1996), Chan (2001), and Ossendrijver et al. (2001). The initial state is in hydrostatic equilibrium but convectively unstable in the upper half of the box. The $z$ coordinate is negative in our setup, with $z=0$ at the upper boundary. The stable layer thus extends from $z=-2$ to $z=-1$, the unstable layer from $z=-1$ to $z=0$. The density varies by a factor 50 over the depth of the box, i.e. the density scale height is 0.5 .

The initial magnetic field is vertical and homogeneous. We run the simulations until a quasi-stationary state evolves. Our control parameters are the heat conduction coefficient, $\kappa$, the Prandtl number, $\operatorname{Pr}=\nu / \kappa$, where $\nu$ is the viscosity, the magnetic diffusivity coefficient, $\eta$, and the strength of the initial magnetic field, $B_{0}$. Convection sets in if the Rayleigh number,

$$
\mathrm{Ra}=\frac{\rho g c_{p} d^{4}}{T \kappa \nu}\left(\frac{d T}{d z}-\frac{g}{c_{p}}\right),
$$

with the density $\rho$, the specific heat capacity $\mathrm{c}_{p}$, the gravity force $g$, and the length scale $d$, exceeds a critical value. The length scale is defined by the depth of the convectively unstable layer, i.e. $d=1$.

\section{Results}

The velocity field shows the asymmetry between upwards and downwards motion characteristic of convection in stratified media. The downward motion is concentrated at the boundaries of the convection cells and particularly at the corners. The upwards motion fills the interior of the convection cells. As it covers a much larger area the gas motion is much slower than in the concentrated downdrafts. The magnetic field shows a similar pattern. The vertical field is concentrated in the areas with downwards motion and weak in the areas with upward motion. As the total vertical magnetic flux is conserved, this is the result of field advection.

Figure 1 shows results from a run with rather weak magnetic field, $B_{0}=10^{-5}$, and $\mathrm{Ra}=6 \times 10^{7}$. The value of the magnetic Prandtl number, $\mathrm{Pm}=\nu / \eta$, is 0.1 . The left diagram shows the horizontal average of the cross helicity as a function of the depth, the right diagram shows the vertical $(z)$ component, $\left\langle u_{z}^{\prime} B_{z}^{\prime}\right\rangle$. The difference between the two quantities is very small, with the vertical component actually being slightly larger than the sum of the vertical and horizontal correlations. It is thus a good proxy for 

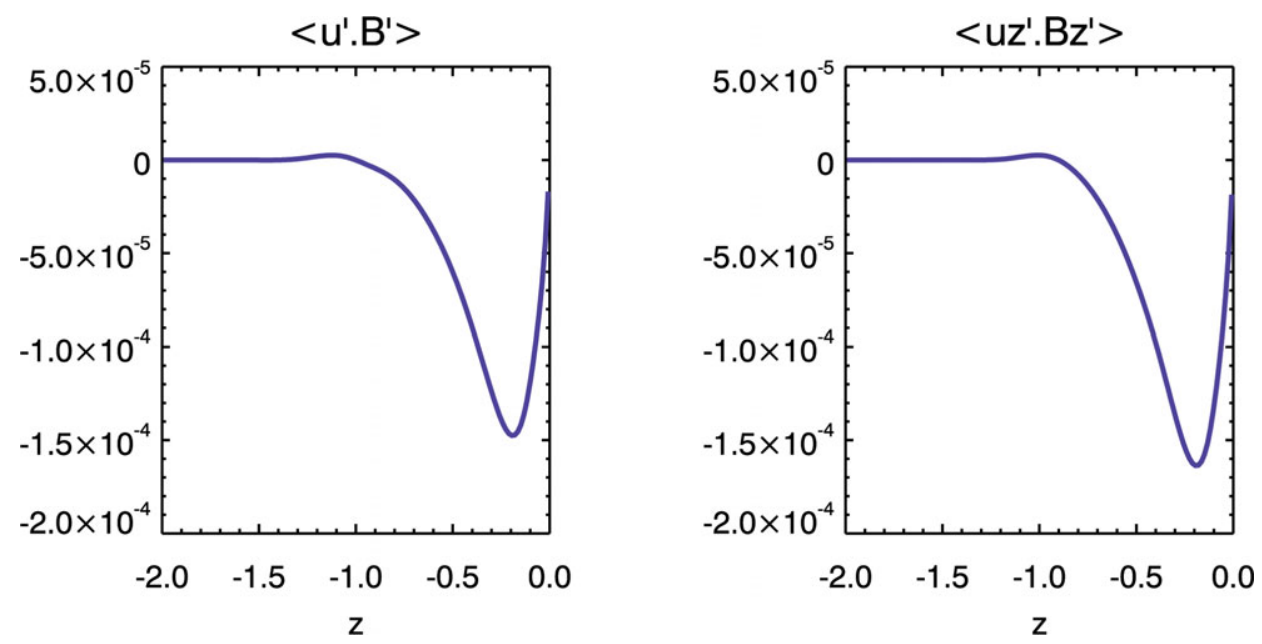

Figure 1. Horizontally-averaged cross helicity as a function of depth. Left: full cross helicity.

Center: correlation of the vertical components of the magnetic field and gas velocity.

the full cross correlation. The correlations do not vanish abruptly at the bottom of the unstable layer because of overshoot, which affects the upper half of the stable layer. The correlations are positive and much smaller than in the unstable layer.

As the density scale height and the magnetic diffusivity are both positive quantities, Eq. 1.3 can only be fulfilled if the (vertical) cross correlation and the mean magnetic field have opposite signs. This is indeed the case. For positive values of the mean magnetic field $\left\langle B_{z}\right\rangle$ the cross-helicity is negative in the unstably stratified layer. If the field polarity is reversed and everything else left unchanged the cross correlation becomes positive with the same amplitude as in the case shown.

\section{References}

Brandenburg, A., Jennings, R. L., Nordlund, A. et al. 1996, J. Fluid Mech., 306, 14370

Cattaneo, F., Brummell, N. H., Toomre, J., et al. 1991, ApJ, 370, 282

Chan, K. L., 2001, ApJ, 548, 1102

Komm, R., Hill, F., \& Howe, R., J. Phys. Conf. Ser., 118, 012035

Ossendrijver, M., Stix, M., Brandenburg, A., 2001, A\& A, 376, 713

Rüdiger, G., Kitchatinov, L. L., \& Brandenburg, A. 2011, Sol. Phys., 30, 490

Ziegler, U. 2002, A\& A, 386,331 